PROCEEDINGS OF THE

AMERICAN MATHEMATICAL SOCIETY

Volume 129, Number 10, Pages 3109-3119

S 0002-9939(01)05915-9

Article electronically published on April 2, 2001

\title{
NAPOLEON IN ISOLATION
}

\author{
DANNY CALEGARI
}

(Communicated by Ronald A. Fintushel)

\begin{abstract}
Napoleon's theorem in elementary geometry describes how certain linear operations on plane polygons of arbitrary shape always produce regular polygons. More generally, certain triangulations of a polygon that tiles $\mathbb{R}^{2}$ admit deformations which keep fixed the symmetry group of the tiling. This gives rise to isolation phenomena in cusped hyperbolic 3-manifolds, where hyperbolic Dehn surgeries on some collection of cusps leave the geometric structure at some other collection of cusps unchanged.
\end{abstract}

\section{Geometric isolation}

1.1. Definition. Let $M$ be a complete, finite volume hyperbolic 3-manifold with $n$ torus cusps, which we denote $c_{1}, \ldots, c_{n}$. The following definition is found in [7]:

Definition 1.1. A collection of cusps $c_{j_{1}}, \ldots, c_{j_{m}}$ is geometrically isolated from a collection $c_{i_{1}}, \ldots, c_{i_{n}}$ if any hyperbolic Dehn surgery on any collection of the $c_{i_{k}}$ leaves the geometric structure on all the $c_{j_{l}}$ invariant.

Note that this definition is not symmetric in the collections $c_{i_{k}}$ and $c_{j_{l}}$, and in fact there are examples which show that a symmetrized definition is strictly stronger (see [7]).

More generally, we can ask for some prescribed set of fillings on the $c_{i_{k}}$ which leave the $c_{j_{l}}$ invariant. Generalized (non-integral) hyperbolic surgeries on a cusp are a holomorphic parameter for the space of all (not necessarily complete) hyperbolic structures on $M$ with a particular kind of allowable singularities (i.e. generalized cone structures) in a neighborhood of the complete structure. Moreover, the complex dimension of the space of geometric shapes on a complete cusp is 1 . Consequently, for dimension reasons whenever $n>m$ there will be families of generalized surgeries leaving the geometric structures on the $c_{j_{l}}$ invariant. There is no particular reason to expect, however, that any of these points will correspond to an integral surgery on the $c_{i_{k}}$. When there is a 1 complex dimensional holomorphic family of isolated generalized surgeries which contains infinitely many integral surgeries, we say that we have an example of an isolation phenomenon.

Neumann and Reid describe other qualities of isolation in [7] including the following:

Definition 1.2. A collection of cusps $c_{j_{1}}, \ldots, c_{j_{m}}$ is strongly isolated from a collection $c_{i_{1}}, \ldots, c_{i_{m}}$ if after any hyperbolic Dehn surgeries on any collection of the $c_{j_{l}}$,

Received by the editors June 15, 1999 and, in revised form, March 6, 2000.

2000 Mathematics Subject Classification. Primary 57M50, 57M25.

(C)2001 American Mathematical Society 
a further surgery on any collection of the $c_{i_{k}}$ leaves the geometry of the (possibly filled) cusps $c_{j_{l}}$ invariant.

A collection of cusps $c_{j_{1}}, \ldots, c_{j_{m}}$ is first-order isolated from a collection $c_{i_{1}}, \ldots, c_{i_{m}}$ if the derivative of the deformation map from generalized surgeries on the $c_{i_{k}}$ to the space of cusp shapes on the $c_{j_{l}}$ vanishes at the complete structure.

By using the structure of the $\Phi$ function defined in [8], Neumann and Reid show that strong isolation and first order isolation are symmetric relations. First order isolation can be restated in terms of group cohomology, and is studied in some papers of Kapovich, notably [5].

In this paper we produce new constructions of isolation phenomena of various qualities, both by extending or modifying known constructions and by introducing a conceptually original construction based on Napoleon's Theorem in plane geometry. The sheer wealth of examples that these techniques can produce, used in combination, strongly suggests that instances of isolation phenomena are not isolated phenomena.

1.2. Holomorphic rigidity. Suppose we want to show that a cusp $d$ is isolated from a cusp $c$. Then since the shape of the complete cusp $d$ depends holomorphically on the generalized surgery on $c$, it suffices to show that infinitely many surgeries on $c$ keep the structure of $d$ fixed, since these can only accumulate at the complete (unfilled) structure, where we know the function relating the structure on $d$ to the surgery on $c$ is regular.

We describe this holomorphic structure in more detail. Choose a meridian $m$ of a cusp $c$. Let $M_{(p, q)}$ be obtained by doing $(p, q)$ surgery on $c$. The hyperbolic structure on $M_{(p, q)}$ determines a representation $\rho_{p, q}: \pi_{1}(M) \rightarrow P S L(2, \mathbb{C})$. The image of $[m]$ under this representation has a well-defined complex length $u$ in $\mathbb{C} / 2 \pi i \mathbb{Z}$, which is the logarithm of the ratio of the eigenvalues of $\rho_{p, q}(m)$. We may choose a branch of the logarithm so that the value 0 corresponds to the complete structure. Then small deformations of the hyperbolic structure on $M$ corresponding to generalized surgeries on $c$ are parameterized in a $2: 1$ way by $u$. The set of Euclidean structures on a complete cusp $d$ are parameterized by the complex orbifold $\mathcal{M}_{1}=\mathbb{H}^{2} / P S L(2, \mathbb{Z})$, and it is well-known that the map from $u \rightarrow \mathcal{M}_{1}$ is analytic and regular at 0 . For more detail, see [8].

1.3. Rigid orbifolds. A systematic study of isolation was initiated in [7]. Most of the examples constructed in [7] of pairs of cusps $c_{1}, c_{2}$ which are geometrically isolated have the property that there is a totally geodesic rigid triangle orbifold separating the two cusps. Such a separating surface splits the manifold $M$ into two pieces $M_{1}, M_{2}$ where $c_{i}$ sits as a cusp in $M_{i}$. Then a surgery on $c_{i}$ deforms only the piece $M_{i}$, keeping the geometry of the splitting orbifold unchanged, and $M_{i}$ can then be glued to $M_{i+1}$ to produce a complete hyperbolic structure on $M$. One sees that the geometry of the entire piece containing the unfilled cusp is unchanged by this operation, and therefore that each of the two cusps is isolated from the other cusp.

If $M$ covers an orbifold $N$ containing a totally geodesic triangle orbifold which separates cusps $c$ and $d$, then in $M$ any surgeries on lifts of $c$ which descend to $N$ will leave unaffected the structures on lifts of $d$.

1.4. Rigid cusps. A refinement of the construction above comes when the rigid orbifold is boundary parallel. The square torus $\mathbb{R}^{2} / \mathbb{Z} \oplus i \mathbb{Z}$ and the hexagonal torus 
$\mathbb{R}^{2} / \mathbb{Z} \oplus \frac{1+\sqrt{3} i}{2} \mathbb{Z}$ have rotational symmetries of order 4 and 6 respectively. If these symmetries about a cusp $c$ can be made to extend over the entire manifold $M$, then any surgery preserving these symmetries will keep the cusp $c$ square or hexagonal respectively. Note that isolation phenomena produced by this method tend to be one-way.

Example 1.1. Let $M=T^{2} \times I-K$ where $K$ is a knot which has 4 -fold rotational symmetry, as seen from either of the $T^{2}$ ends of $T^{2} \times I$. Then $M$ has 4 -fold rotational symmetry which is preserved after $(p, q)$ filling on the knot $K$. This symmetry keeps the shape of the two ends of $T^{2} \times I$ square after every surgery on $K$. In general, surgery on either of the ends of the $T^{2}$ will disrupt the symmetry and not exhibit any isolation.

1.5. Mutation. An incompressible surface in a hyperbolic manifold does not need to be rigid for certain topological symmetries to be realized as geometric symmetries. In a finite volume hyperbolic 3 -manifold, an incompressible, $\partial$-incompressible surface $S$ without accidental parabolics which is not the fiber of a fibration over $S^{1}$ is quasifuchsian, and therefore corresponds to a unique point in Teich $(S) \times T e i c h(\bar{S})$. For surfaces $S$ of low genus, the tautological curve over the Teichmüller space of $S$ has certain symmetries which restrict to a symmetry of each fiber - that is, of each Riemann surface topologically equivalent to $S$. There is a corresponding symmetry of the universal curve over Teich $(\bar{S})$ and therefore of a quasifuchsian representation of $S$. Geometrically, this means that one can cut along a minimal surface representing the class of $S$ and reglue it by an automorphism which preserves the geometric structure to give a complete nonsingular hyperbolic structure on a new manifold. Actually, one does not need to know there is an equivariant minimal surface along which one can cut - one can perform the cutting and gluing at the level of limit sets by using Maskit's combinations theorems ([] $]$. This operation is called mutation and is studied extensively by Ruberman, for instance, in [10].

If $S$ is a sphere with 4 punctures, we can think of $S$ as a Riemann sphere with 4 points deleted. After a Möbius transformation, we can assume 3 of these points are at $0,1, \infty$ and the 4 th is at the complex number $z$. The full symmetry group $\mathrm{S}_{4}$ does not act holomorphically on $S$ except in very special cases, but the subgroup consisting of (12)(34) and its conjugates does act holomorphically. This group is known as the Klein 4-group.

Geometrically, where we find an appropriate 4-punctured sphere in a hyperbolic manifold $M$, we can cut along the sphere and reglue after permuting the punctures. This operation leaves invariant the geometric structure on those pieces of the manifold that do not meet the sphere. If $S$ is a spherical orbifold or cone manifold with 4 equivalent cone points, we can similarly cut and reglue. The sphere along which the mutation is performed is called a Conway sphere. The observation that mutation can be performed for spherical orbifolds is standard: one can always find a finite manifold cover of any hyperbolic orbifold, by Selberg's lemma. The spherical orbifold lifts to an incompressible surface in this cover, and one can cut and reglue equivariantly in the cover. In fact, local rigidity for small cone angles developed by Hodgson and Kerckhoff in [4] suggests that this can be done just as easily for the cone manifold case, but this is superfluous for our applications.

Example 1.2. Let $M$ be a manifold with a single cusp $c$ which admits a $\mathbb{Z} / 3 \mathbb{Z}$ symmetry that acts on $c$ as a rotation. This symmetry forces $c$ to be hexagonal. Let $p$ be a point in $M$ not fixed by $\mathbb{Z} / 3 \mathbb{Z}$, and let $M^{\prime}$ be obtained by equivariantly 
removing 3 balls centered at the orbit of $p$. Glue another manifold with $\mathbb{Z} / 3 \mathbb{Z}$ symmetry to $M^{\prime}$ along its spherical boundaries $S_{1}, S_{2}, S_{3}$ so that the symmetries on either side are compatible, to make $M^{\prime \prime}$. Let $K$ be a $\mathbb{Z} / 3 \mathbb{Z}$-invariant knot in $M^{\prime \prime}$ which intersects each of the spheres $S_{i}$ in 4 points and which is sufficiently complicated that its complement in $M^{\prime \prime}$ is atoroidal and the 4-punctured spheres $S_{i}$ are incompressible and $\partial$-incompressible. Perform a mutation on $S_{1}$ which destroys the $\mathbb{Z} / 3 \mathbb{Z}$-symmetry to get a manifold $N$ with two cusps which we refer to as $c$ and $d$, where $d$ corresponds to the core of $K$. Then $c$ is hexagonal, since mutation does not affect the geometric structure away from the splitting surface. Moreover, for large integers $r$, any generalized $(r, 0)$ surgery on $d$ will preserve the fact that the $S_{i}$ are incompressible, $\partial$-incompressible spheres with 4 cone points of order $r$, and therefore we can undo the mutation on $S_{1}$ to see that these surgeries do not affect the hexagonal structure on $c$. But if a real 1-parameter family of surgeries on $d$ does not affect the structure on $c$, then every surgery on $d$ keeps the structure on $c$ fixed, so $c$ is isolated from $d$. Note that for general $(p, q)$ surgeries on $d$, the spheres $S_{i}$ will be destroyed and the surgered manifold will not be mutation-equivalent to a $\mathbb{Z} / 3 \mathbb{Z}$ symmetric manifold. Moreover, for sufficiently generic $K$, there will be no rigid triangle orbifolds in $N$ separating $c$ from $d$. As far as we know, this is the first example of geometric isolation to be constructed that is not forced by a rigid separating or boundary parallel surface.

We note in passing that mutation followed by surgery often preserves other analytic invariants of hyperbolic cusped manifolds, such as volume and (up to a constant) Chern-Simons invariants. Ruberman observed in 10 that if $K$ and a Conway sphere $S$ are unlinked, then a mutation which preserves this unlinking can actually be achieved by mutation along the genus 2 surface obtained by tubing together the sphere $S$ with a tubular neighborhood of $K$. This mutation corresponds to the hyperelliptic involution of a genus 2 surface. Since this surface is present and incompressible for all but finitely many surgeries on $K$ and its mutant, the surgered mutants are mutants themselves. If $S$ and $K$ are not unlinked, no such hyperelliptic surface can be found, and in fact in this case there is no clear relationship between invariants of the manifold obtained after surgeries on the original knot and on the mutant. Perhaps this makes the persistence of isolation under mutation more interesting, since it shows that some of the effects of mutation are persistent under surgeries where other effects are destroyed.

\section{NAPOLEON'S THEOREM}

2.1. Triangulations and hyperbolic surgery. A finite volume complete but not compact hyperbolic 3-manifold $M$ can be decomposed into a finite union of (possibly degenerate) ideal tetrahedra glued together along their faces. An ideal tetrahedron determines and is determined by its fourtuple of endpoints on the sphere at infinity of $\mathbb{H}^{3}$. Identifying this sphere with $\mathbb{C P}^{1}$, we can think of the ideal tetrahedron as a 4 -tuple of complex numbers. The isometry type of the tetrahedron is determined by the cross-ratio of these 4 points; equivalently, if we move three of the points to $0,1, \infty$ by a hyperbolic isometry, the isometry type of the tetrahedron is determined by the location of the 4th point, that is by a value in $\mathbb{C}-\{0,1\}$. This value is referred to as the simplex parameter of the ideal tetrahedron. A combinatorial complex $\Sigma$ of simplices glued together can be realized geometrically as an ideal triangulation of a finite volume hyperbolic manifold (after 
removing the vertices of $\Sigma$ ) if certain equations in the simplex parameters are satisfied. These equations can be given explicitly by examining the links of vertices of the triangulation.

For a finite volume non-compact hyperbolic manifold, all the links of vertices of $\Sigma$ should be tori $T_{j}$. There are induced triangulations $\tau_{j}$ of these tori by the small triangles obtained by cutting off the tips of the tetrahedra in $\Sigma$. Let $\Sigma=\bigcup_{i} \Delta_{i}$ and let $z_{i}$ be a hypothetical assignment of simplex parameters to the tetrahedra $\Delta_{i}$. Then the horoball sections of a hypothetical hyperbolic structure on $\Sigma^{\prime}=$ $\Sigma$ - vertices are Euclidean tori triangulated by Euclidean triangles, which are the horoball sections of the $\Delta_{i}$. If the simplex parameter of an ideal tetrahedron is $z$, the Euclidean triangles obtained as sections of the horoballs centered at its vertices have the similarity type of the triangle in $\mathbb{C}$ with vertices at $0,1, z$. A path in the dual 1-skeleton to $\tau_{j}$ determines a developing map of $\tau_{j}$ in $\mathbb{C}$ : given a choice of the initial triangle, there is a unique choice of each subsequent triangle within its fixed Euclidean similarity type such that the combinatorial edge it shares with its predecessor is made to geometrically agree with it. The holonomy of a closed path in this dual skeleton is the Euclidean similarity taking the initial triangle to the final triangle.

There are two necessary conditions to be met in order for the hyperbolic structures on the $\Delta_{i}$ to glue up to give a hyperbolic structure on $\Sigma^{\prime}$. These conditions are actually sufficient in a small neighborhood of the complete structure as described in [8] and made rigorous in [9]:

- The edge equations: the holonomy around a vertex of $\tau_{j}$ should be trivial.

- The cusp equations: the holonomy around the meridian and longitudes of the $T_{j}$ should be translations.

These "equations" can be restated as identities of the form

$$
\sum_{i} c_{i j} \ln \left(z_{i}\right)+d_{i j} \ln \left(1-z_{i}\right)=\pi i e_{j}
$$

for some collection of integers $c_{i j}, d_{i j}, e_{j}$ and some appropriate choices of branches of the logarithms of the $z_{i}$.

A $(p, q)$ hyperbolic Dehn surgery translates in this context to replacing the cusp equations for some cusp by the condition that the holonomy around the meridian and longitude give Euclidean similarities $h_{m}, h_{l}$ such that $h_{m}^{p} h_{l}^{q}=\mathrm{id}$. The analytic co-ordinates on Dehn surgery space determined by the analytic parameters $z_{i}$ are holomorphically related to the $u$ co-ordinates alluded to earlier. The geometry of a complete cusp is determined by the translations corresponding to the holonomy of the meridian and longitude.

This is all described in great detail in [8] and [9].

2.2. Tessellations with forced symmetry. In this section, we produce examples of isolation phenomena which do not come from rigidity or mutation, but rather from the following theorem, known as "Napoleon's Theorem" ([3]):

Theorem 2.1 ("Napoleon's Theorem"). Let $T$ be a triangle in $\mathbb{R}^{2}$. Let $E_{1}, E_{2}, E_{3}$ be three equilateral triangles constructed on the sides of $T$. Then the centers of the $E_{i}$ form an equilateral triangle.

Proof. Let the vertices of $T$ be $0,1, z \in \mathbb{C}$. The three centers of the $E_{i}$ are of the form $a_{i} z+b_{i}$ for certain complex numbers $a_{i}, b_{i}$ so the shape of the resulting triangle 
is a holomorphic function of $z$. Let $\omega=\left(1+\frac{i}{\sqrt{3}}\right) / 2$. If $z$ is real and between 0 and 1 , then the center of $E_{1}$ splits the line between 0 and $\omega$ in the ratio $z, 1-z$, the center of $E_{2}$ splits the line between $\omega$ and 1 in the ratio $z, 1-z$, and the center of $E_{3}$ is at $1-\omega$. But a clockwise rotation through $\pi / 3$ about $1-\omega$ takes the line between 0 and $\omega$ to the line between $\omega$ and 1; i.e. it takes the center of $E_{1}$ to the center of $E_{2}$. Thus the theorem is true for real $z$ and by holomorphicity it is true for all $z$.

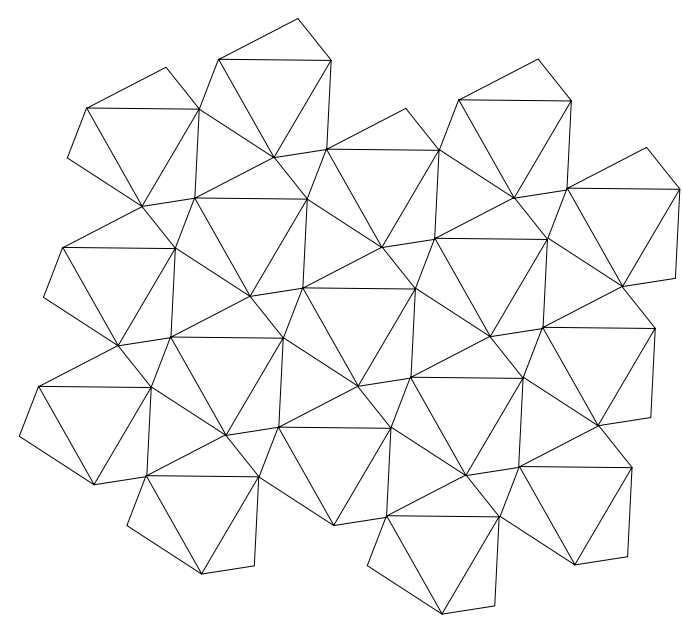

Figure 1. Three isometric triangles and three equilateral triangles make up a hexagon which tiles the plane with symmetry group $\mathrm{S}(3,3,3)$

Napoleon's Theorem gives rise to an interesting phenomenon in plane geometry: fix a triangle $T$. Then three triangles isometric to $T$ and three equilateral triangles with side length equal to the sides of $T$ can be glued together to make a hexagon which tiles the plane with symmetry group $\mathrm{S}(3,3,3)$ as in Figure 1 . The edge lengths of the three equilateral triangles are equal to the three edge lengths of $T$, and around each vertex the angles are the three interior angles of $T$ together with three angles equal to $\frac{2 \pi}{6}$. It follows that the hexagon in question exists and tiles the plane; to see that it has the purported symmetry group, observe that the combinatorics of the triangulation have a 3-fold symmetry about the centers of the three equilateral triangles bounding some fixed triangle of type $T$. These three centers are the vertices of an equilateral triangle, by Napoleon's Theorem; it follows that the symmetry group of the tiling contains the group generated by three rotations of order 3 with centers at the vertices of an equilateral triangle that is to say, the symmetry group $\mathrm{S}(3,3,3)$.

If we imagine that these triangles are the asymptotic horoball sections of ideal tetrahedra going out a cusp of a hyperbolic manifold, we see that appropriate deformations of the tetrahedral parameters change the triangulation but not the geometry of the cusp. For $T$ a horoball section of an ideal tetrahedron with simplex parameter $z$, the holonomy around vertices is just

$$
z \cdot \omega \cdot \frac{z-1}{z} \cdot \omega \cdot \frac{1}{1-z} \cdot \omega=1
$$


where $\omega=\frac{1+i \sqrt{3}}{2}$ is the similarity type of an equilateral triangle, and the holonomy around the meridian and longitude are both translations by complex numbers $z_{1}, z_{2}$ whose ratio is $\frac{1+i \sqrt{3}}{2}$.
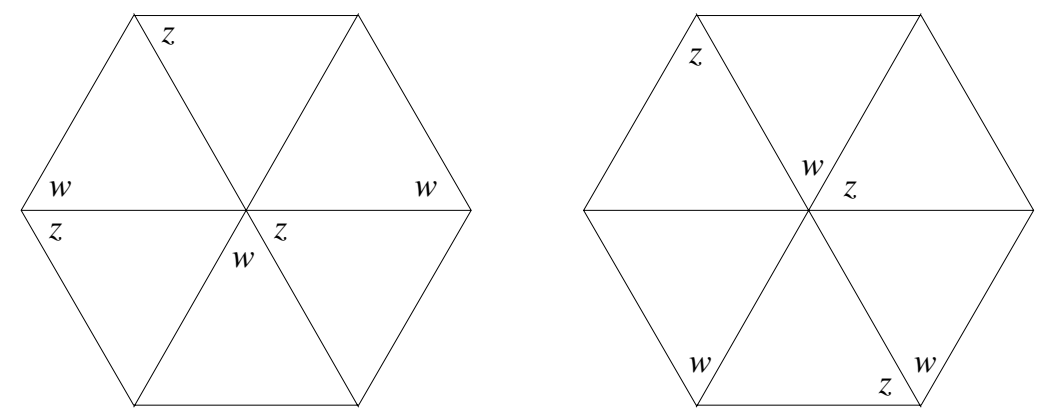

Figure 2. Two cusps with triangle parameters related as indicated in the diagram can be deformed independently through (incomplete) affine structures. We follow the conventions of [8], so that if the vertices of $\Delta$ are at $\{0,1, \infty, z\}$, the edge marked with $z$ runs from 0 to $\infty$ or from 1 to $z$.

Example 2.1. A scheme to parlay this theorem into isolation phenomena is given by the following setup: we have two hexagons $H_{1}, H_{2}$ each tiled by six equilateral triangles. We divide these twelve triangles into two similarity types, corresponding to the triangles in $\mathbb{C}$ with vertices at $\{0,1, z\}$ and $\{0,1, w\}$, and we choose a preferred vertex for each triangle corresponding to 0 , in the manner indicated in Figure 2. For an arbitrary choice of complex numbers $z$ and $w$, the hexagons can be realized geometrically to give affine structures on the tori obtained by gluing opposite sides of $H_{1}$ together and similarly for $H_{2}$. Initially set $z=w=\frac{1+i \sqrt{3}}{2}$. Deforming $z$ but keeping $w$ fixed changes the affine structure on the first torus but leaves the structure on the second torus unchanged. The combinatorial triangulation of the second torus is exactly the triangulation of a fundamental domain of the tessellation in Figure 1. It follows that for $w=\frac{1+i \sqrt{3}}{2}$ and $z$ arbitrary, the universal cover of the second torus is tiled by a tessellation with symmetry group $\mathbf{S}(3,3,3)$ and the torus is therefore hexagonal.

Similarly, deforming $w$ and keeping $z=\frac{1+i \sqrt{3}}{2}$ changes the affine structure on the second torus but leaves the structure on the first torus unchanged, since now we can identify the combinatorial triangulation of the first torus with the triangulation of a fundamental domain of the tessellation in Figure 1.

This configuration of ideal tetrahedra with horoball sections equal to the two cusps in this figure can be realized geometrically by arranging six regular ideal tetrahedra in the upper-half space in a hexagonal pattern with the common edge of the tetrahedra going from 0 to $\infty$. The pattern seen from infinity looking down is $H_{1}$, and the pattern seen from 0 looking up is $H_{2}$. The pictures are aligned so that the real line has its usual orientation. Glue the twelve free faces of the tetrahedra in such a way as to make $H_{1}$ and $H_{2}$ torus cusps. This gives an orbifold $N$ whose underlying manifold is $T^{2} \times I$, with orbifold locus three arcs of cone angle $2 \pi / 3$ each running between two $(3,3,3)$ triangle cusps arranged in the obvious symmetrical manner. 
Unfortunately, under surgeries of the cusps of $N$, the simplices do not deform in the manner required by Napoleon's Theorem. However, if we pass to a 3-fold cover, this problem can be corrected.

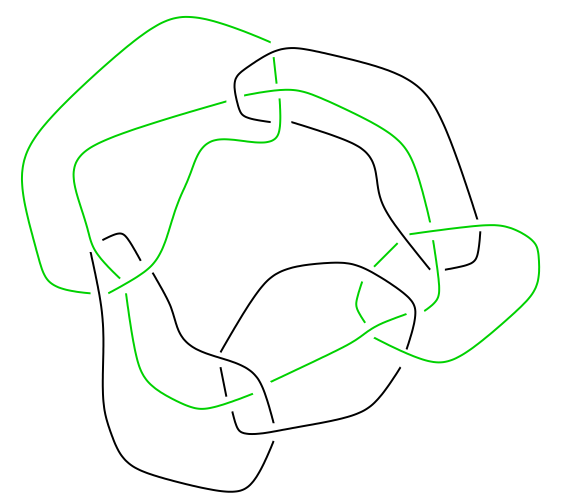

FiguRE 3. "Napoleon's 3-manifold" has a decomposition into ideal tetrahedra such that surgery on a "dark" cusp deforms the triangulation of the other "dark" cusps but keeps the shape of the cusp constant, as guaranteed by Napoleon's Theorem. A similar relation holds for the "light" cusps.

Let $L$ be the link depicted in Figure 3, and let $M=S^{3}-L$. Then $M$ admits a complete hyperbolic structure which can be decomposed into 18 regular ideal tetrahedra. It follows that $M$ is commensurable with the figure 8 knot complement. In fact, $M$ is the 3 -fold cover of $N$ promised above.

Geometrically, arrange 18 regular ideal tetrahedra in the upper half-space with a common vertex at infinity so that a horoball section intersects the collection the pattern depicted in Figure 4. If we glue the 12 external vertical sides of this collection of tetrahedra in the indicated manner, it gives a horoball section of one of the cusps of $M$. It remains to glue up the 18 faces of the ideal tetrahedra with all vertices on $\mathbb{C}$. Figure 4 has an obvious decomposition into 3 regular hexagons, each composed of 6 equilateral triangles. The 3 edges of the complex associated to the centers of these three hexagons have a common endpoint at $\infty$ and intersect $\mathbb{C}$ at three points $p_{1}, p_{2}, p_{3}$. Six triangles come together at each of the points $p_{i}$, and a horoball centered at each of these points intersects 6 tetrahedra in a hexagonal pattern. Glue opposite faces of these hexagons to produce 3 cusps centered at $p_{1}, p_{2}, p_{3}$. The result of all this gluing produces the manifold $M$. By construction, this particular choice of triangulation produces 6 hexagonal cusps, 3 made up of 6 equilateral triangles and 3 made up of 18 equilateral triangles.

The components of $L$ fall into two sets of 3 links, depicted in the figure as the darker and the lighter links, which we denote by $c_{1}, c_{2}, c_{3}$ and $d_{1}, d_{2}, d_{3}$ respectively. The group of symmetries of $M$ permutes the cusps by the group $\left(\mathrm{S}_{3} \times \mathrm{S}_{3}\right) \rtimes \mathbb{Z} / 2 \mathbb{Z}$ where the conjugation action of the generator of $\mathbb{Z} / 2 \mathbb{Z}$ takes $\left(\sigma_{i}, \sigma_{j}\right)$ to $\left(\sigma_{j}, \sigma_{i}\right)$. If $G$ is the entire group of symmetries of $M$, then there is a short exact sequence

$$
0 \longrightarrow \mathbb{Z} / 3 \mathbb{Z} \longrightarrow G \longrightarrow\left(\mathrm{S}_{3} \times \mathrm{S}_{3}\right) \rtimes \mathbb{Z} / 2 \mathbb{Z} \longrightarrow 0
$$

so the order of $G$ is 216 . 


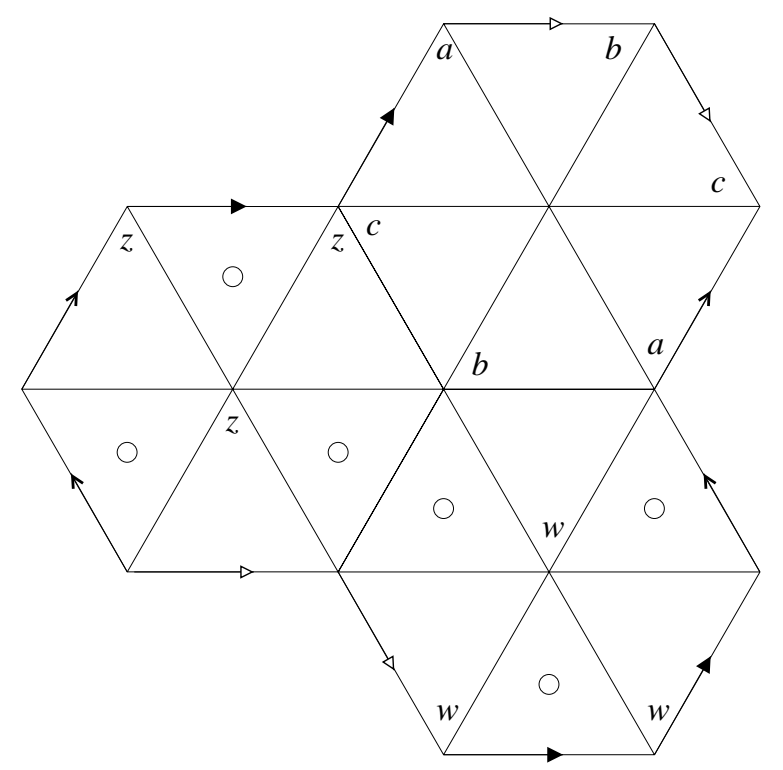

FiguRE 4. A horoball section of the cusp $d_{1}$ of Napoleon's 3manifold. When Dehn surgery is performed on $c_{1}$, the triangles making up $d_{1}$ deform in the manner indicated. The triangles marked with a circle stay equilateral under the deformation.

These links have the property that a surgery on $c_{i}$ keeps $c_{i+1}$ and $c_{i+2}$ hexagonal, but distorts the structure at the $d_{j}$, and vice versa. On the other hand, a surgery on both $c_{i}$ and $c_{i+1}$ does distort the structure at $c_{i+2}$. In terms of the picture already described, the configuration of 18 triangles in Figure 4 decomposes into 3 hexagons of 6 triangles. These three hexagons glue up in the obvious way to give hexagonal triangulations of the $c_{i}$. Under surgery on $c_{i}$, the tetrahedra intersecting $c_{i}$ deform to satisfy the new modified cusp equations. Under this deformation, the other triangle types must deform to keep the other cusps complete. It can be easily checked that the triangulations of the cusps $c_{i+1}, c_{i+2}$ lift to the symmetric tessellations of $\mathbb{R}^{2}$ depicted in Figure 1, and therefore the similarity types of these cusps stay hexagonal. However, under surgery on both $c_{i}$ and $c_{i+1}$, the similarity types of triangles making up cusp $c_{i+2}$ are not related in any immediately apparent way to the picture in Figure 1. The proof that $c_{2}$ and $c_{3}$ are isolated from $c_{1}$ is essentially just a calculation that under surgery on $c_{1}$ say, the simplex parameters solving the relevant edge and cusp equations for the combinatorial triangulation vary as indicated in Figure 4. One may check experimentally that the similarity type of $c_{3}$ is not constant under fillings on both $c_{1}$ and $c_{2}$, using for example Jeff Weeks' program snappea, available from [11], for finding hyperbolic structures on 3-manifolds, or Andrew Casson's provably accurate program cusp (2]).

Definition 2.1. Say that 3 cusps are in Brunnian isolation when a surgery on one of them leaves invariant the structure at the other 2, but a surgery on two of the cusps can change the structure of the third.

With this definition, we observe that Napoleon's 3-manifold has two sets of cusps in Brunnian isolation. 
One can see that there is an automorphism of $S^{3}$ of order 2 fixing two components of $L$ and permuting the other 4 components in pairs. The quotient by this automorphism is an orbifold $N$ which has two regular cusps and two pillow cusps. We call the pillow cusps $c_{p}, d_{p}$ and the regular cusps $c_{r}, d_{r}$ where $c_{p}$ is a quotient of $c_{1}, c_{r}$ is covered by $c_{2} \cup c_{3}$, and similarly for the $d_{i}$.

The cusp $c_{p}$ is first-order isolated but not isolated from $c_{r}$. Similarly, $d_{p}$ is firstorder isolated but not isolated from $d_{r}$. This can easily be observed by noting that $c_{r}$ is isolated from $c_{p}$, and therefore it is first-order isolated from it (by the corresponding properties in the cover). It follows that $c_{p}$ is first-order isolated from $c_{r}$ by [7]. To see that $c_{p}$ is not isolated from $c_{r}$, it suffices to pass to the cover and perform an equivariant surgery there. Alternatively, one can easily check by hand using snappea that the geometry of the cusp $c_{p}$ changes when one performs surgery on $c_{r}$.

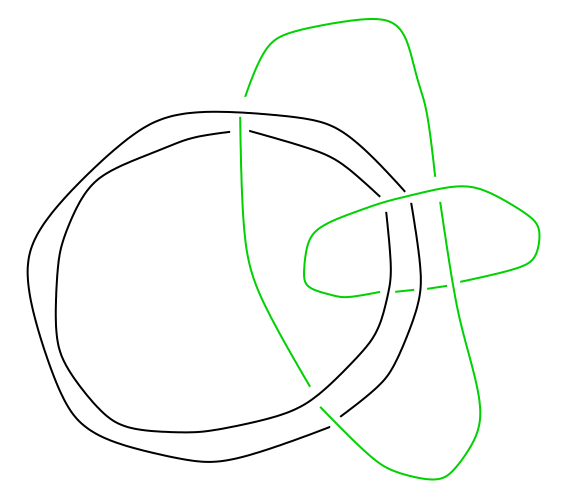

Figure 5. A link in $S^{3}$ whose complement displays Napoleonic tendencies

Example 2.2. The 2-cusped orbifold $A$ first described in [7] and studied in [1] displays Napoleonic tendencies, where the version of Napoleon's theorem we use now concerns right triangles. It is obtained by $(2,0)$ surgery on the light cusps of the link complement portrayed in Figure 5. Coincidentally, it is 2-fold covered by that very link complement. Let $T$ be the right triangle with side lengths $\{1,1, \sqrt{2}\}$, and let $S$ be the unit square in $\mathbb{C}$. Pick a point $p \in S$ and construct four triangles $T_{i}$ all similar to $T$ with one vertex of the diagonal at $p$ and the other vertex of the diagonal at a vertex of $S$, such that the triangle is clockwise of the diagonal, seen from $p$. Then the 8 vertices of these triangles away from $p$ are the vertices of an octagon which tiles the plane with quotient space the square torus. This corresponds exactly to the triangulation of a horoball section of a complete cusp in the orbifold $A$ after a deformation of the other cusp. One observes that the link complement in Figure 3 is obtained from the link complement in Figure 5 by drilling out two curves. Are the isolation phenomena associated with the two links related?

\section{REFERENCES}

1. D. Calegari, A note on strong geometric isolation in 3-orbifolds, Bull. Aust. Math. Soc. $\mathbf{5 3} 2$ (1996) pp. 271-280 MR 97e:57017

2. A. Casson, cusp computer program. 
3. H. Coxeter and S. Greitzer, Geometry revisited, MAA New Math. Library 19 (1967)

4. C. Hodgson and S. Kerckhoff, Rigidity of hyperbolic cone-manifolds and hyperbolic Dehn surgery, J. Diff. Geom. 48 no. 1 (1998) pp. 1-59 MR 99b:57030

5. M. Kapovich, Eisenstein series and Dehn surgery, MSRI preprint (1992)

6. B. Maskit, Kleinian groups, Springer-Verlag (1987) MR 90a:30132

7. W. Neumann and A. Reid, Rigidity of cusps in deformations of hyperbolic 3-orbifolds, Math. Ann. 295 (1993) pp. 223-237 MR 94c:57025

8. W. Neumann and D. Zagier, Volumes of hyperbolic 3-manifolds, Topology 243 (1985) pp. 307-332 MR 87j:57008

9. C. Petronio and J. Porti, Negatively oriented ideal triangulations and a proof of Thurston's hyperbolic Dehn filling theorem, math.GT/9901045

10. D. Ruberman, Mutation and volumes of knots in $S^{3}$, Invent. Math. 901 (1987) pp. 189-215 MR 89d:57018

11. J. Weeks, snappea computer program available from http://thames.northnet.org/weeks/ index/SnapPea.html

Department of Mathematics, University of California, Berkeley, California 94720

E-mail address: dannyc@math.berkeley.edu

Current address: Department of Mathematics, Harvard University, Cambridge, Massachusetts 02138

E-mail address: dannyc@math.harvard.edu 